\title{
On Approximation of Average Expectation Prices for Path Dependent Options in Fractional Models ${ }^{\star}$
}

\author{
Bartosz Ziemkiewicz \\ Faculty of Mathematics and Computer Science, \\ Nicolaus Copernicus University, \\ ul. Chopina 12/18, 87-100 Toruń, Poland \\ bartek@mat.uni.torun.pl
}

\begin{abstract}
Using integral representation of a fractional Brownian motion $(\mathrm{fBm})$ we propose a new method of approximation of its trajectories as well as trajectories of a geometric $\mathrm{fBm}$. We give the rate of convergence in $\mathbb{L}^{p}$-norm of the approximation. Applications to pricing of path dependent options in a fractional model are considered. Lookback and Asian options are examined in detail.
\end{abstract}

\section{Introduction}

Let $\left(B_{t}^{\mathbb{H}}\right)_{t \in[0, T]}$ be a fractional Brownian motion (fBm) with Hurst index $\mathbb{H} \in(1 / 2,1)$, i.e. $B^{\mathbb{H}}$ is a centered Gaussian process with covariance $E B_{t}^{\mathbb{H}} B_{s}^{\mathbb{H}}=$ $\frac{1}{2}\left(t^{2 \mathbb{H}}+s^{2 \mathbb{H}}-|t-s|^{2 \mathbb{H}}\right)$ defined on some probability space $(\Omega, \mathcal{F}, P)$. We denote by $\left(\mathcal{F}_{t}\right)_{t \in[0, T]}$ the filtration generated by $\left(B_{t}^{\mathbb{H}}\right)$. We consider a continoustime market model with two assets, the riskless bond $\left(B_{t}\right)_{t \in[0, T]}$, and the risky stock $\left(S_{t}\right)_{t \in[0, T]}$. The dynamics for the prices are

$$
B_{t}=e^{r t}, \quad S_{t}=S_{0} e^{\mu t+\sigma B_{t}^{\mathbb{H}}},
$$

where $S_{0}>0$ and $\mu \in \mathbb{R}, r, \sigma>0$. It is convenient to make the assumption that there are no dividends, and no transaction costs. An option will be any non-negative $\mathcal{F}_{T}$-measurable random variable $f_{T}$.

Note that if $\mathbb{H}=\frac{1}{2}$, the above model coincides with the well known BlackScholes model. In this case a fair price of the option is the discounted expectation of $f_{T}$ with respect to the martingale measure of this model.

If $\mathbb{H} \neq \frac{1}{2}$, then $B^{\mathbb{H}}$ is not a semimartingale, and martingale measures do not exist (see e.g. 4]), so we need a different method for option pricing. Instead of martingale measures we will use the so-called average risk neutral measures.

Definition 1 (6]). If the measure $Q$ is equivalent to the measure $P$, and

$$
E^{Q}\left(S_{t}\right)=e^{r t}
$$

for all $t \in[0, T]$ then $Q$ is called the average risk neutral measure.

Existence and uniqueness of $Q$ was proved by Valkeila [6].

\footnotetext{
* Research supported by Komitet Badań Naukowych under grant 1 P03A 02226
} 
Definition 2. The average expectation price $C\left(f_{T}\right)$ of option $f_{T}$ is defined by

$$
C\left(f_{T}\right)=e^{-r T} E^{Q} f_{T} .
$$

If $\mathbb{H}=\frac{1}{2}$, the price $C\left(f_{T}\right)$ coincides with the classical one for the Black-Scholes model. Definition 2 extends the one given by Valkeila [6], where $C\left(f_{T}\right)$ is defined for options depending only on the price of $S$ at the expiration time $T$, i.e. $f_{T}=$ $h\left(S_{T}\right)$ for some $h: \mathbb{R}^{+} \rightarrow \mathbb{R}^{+}$. In this case we have the following explicit formula

$$
C\left(f_{T}\right)=e^{-r T} \frac{1}{\sqrt{2 \pi}} \int_{-\infty}^{+\infty} h\left(S_{0} \exp \left\{\sigma y T^{\mathbb{H}}+r T-\frac{\sigma^{2}}{2} T^{2 \mathbb{H}}\right\}\right) e^{-\frac{y^{2}}{2}} d y .
$$

In the present paper we focus on path dependent options, i.e. options whose payoffs depend on the whole path of $S$. For example, the payoff of a lookback option depends on the minimum or maximum price of $S$, while the payoff of an Asian option depends on the average price of $S$ during the life of option. Evaluation of path dependent options in fractional models is difficult and analytic formulas like (2) are not known. In the paper we will estimate $C\left(f_{T}\right)$ using the Monte Carlo method.

\section{Approximation of $\mathrm{fBm}$}

In the paper we introduce a new method of approximating of $B^{\mathbb{H}}$ based on the integral representation of $\mathrm{fBm}$ as provided in [2. Define the so-called fundamental martingale $\left(M_{t}\right)$ by

$$
M_{t}=\frac{c_{\mathbb{H}}}{2 \mathbb{H}} \int_{0}^{t} s^{1 / 2-\mathbb{H}} d W_{s},
$$

where $c_{\mathbb{H}}$ is a constant depending on $\mathbb{H}$ and $W$ is a standard Brownian motion. $M$ is a Gaussian martingale with independent increments. Now, if we put

$$
Y_{t}=2 \mathbb{H} \int_{0}^{t}(t-s)^{\mathbb{H}-1 / 2} d M_{s}
$$

then the process

$$
B_{t}^{\mathbb{H}}=\int_{0}^{t} s^{\mathbb{H}-1 / 2} d Y_{s}
$$

is a fractional Brownian motion (for the proof we refer to [2]).

To implement this method we proceed as follows.

1. Let $M^{n}$ be a discretization of $M$, i.e. $M_{t}^{n}=M_{\frac{k}{n}}$ for $t \in\left[t_{k}, t_{k+1}\right)$, where $t_{k}=\frac{k}{n}, k=0,1, \ldots,[n T]$. We can simulate the increments of $M_{t}^{n}$ using the fact that $M_{t_{k}}^{n}-M_{t_{k-1}}^{n} \sim \mathcal{N}\left(0, C\left(t_{k}^{2-2 \mathbb{H}}-t_{k-1}^{2-2 \mathbb{H}}\right)\right)$. 
2. Next, we define the approximation $Y^{n}$ of $Y$ by

$$
Y_{t}^{n}=2 \mathbb{H} \sum_{t_{k} \leq t^{*}}\left(t^{*}-t_{k-1}\right)^{\mathbb{H}-1 / 2}\left(M_{t_{k}}^{n}-M_{t_{k-1}}^{n}\right),
$$

where $t^{*}=\max \left\{t_{k} ; t_{k} \leq t\right\}$. It is worth noting that $\left(t^{*}-t_{k}\right)^{\mathbb{H}-1 / 2}$ can be written in the form $\left(\frac{m}{n}\right)^{\mathbb{H}-1 / 2}$, where $m, n \in \mathbb{N}, m \leq[n T]$, so to compute $Y^{n}$ it is sufficient to compute $m^{\mathbb{H}-1 / 2}$ for $m=1, \ldots,[n T]$.

3. Finally, we approximate $B^{\mathbb{H}}$ by sums of the form

$$
B_{t}^{n}=\sum_{t_{k} \leq t^{*}} t_{k-1}^{\mathrm{H}-\frac{1}{2}}\left(Y_{t_{k}}^{n}-Y_{t_{k-1}}^{n}\right) .
$$

Let $\|\cdot\|_{p}$ denotes the usual $\mathbb{L}^{p}$-norm.

Theorem 1. Let $\left(B_{t}^{n}\right)$ be a process defined by (4). Then for any $p \geq 1, \epsilon>0$ and $T \in \mathbb{R}^{+}$

$$
\left\|\sup _{t \leq T}\left|B_{t}^{\mathbb{H}}-B_{t}^{n}\right|\right\|_{p}=\mathcal{O}\left(\frac{1}{n^{\mathbb{H}^{*}-\epsilon}}\right),
$$

where $\mathbb{H}^{*}=\min (1-\mathbb{H}, \mathbb{H}-1 / 2)$.

Proof. From (3) it follows that $a_{t}=[M]_{t}=C t^{2-2 \mathbb{H}}$, where $C$ is a constant depending on $\mathbb{H}$. Hence $M_{t}=\bar{W}_{a_{t}}$ where $\bar{W}$ is a standard Brownian motion. Using the known estimates on the modulus of continuity of $\bar{W}$ (see e.g. [5]) we get

$$
\begin{aligned}
\left\|\sup _{t \leq T}\left|M_{t}-M_{t}^{n}\right|\right\|_{p} & \leq\left\|\omega_{M}(1 / n, T)\right\|_{p}=\left\|\omega_{\bar{W}_{a}}(1 / n, T)\right\|_{p} \\
& \leq\left\|\omega_{\bar{W}}(a(1 / n), T)\right\|_{p} \leq \operatorname{Const}\left(a(1 / n) \ln \left(a(1 / n)^{-1}\right)\right)^{1 / 2} \\
& \leq \operatorname{Const} \frac{\sqrt{\ln n}}{n^{1-\mathbb{H}}}
\end{aligned}
$$

where $\omega_{x}(\delta, T)=\sup \left\{\left|x_{t}-x_{s}\right| ; s, t \in[0, T],|s-t| \leq \delta\right\}, \delta>0, T \in \mathbb{R}^{+}$, $x \in C\left([0, T], \mathbb{R}^{+}\right)$.

We next turn to estimating $\left\|\sup _{t \leq T}\left|Y_{t}-Y_{t}^{n}\right|\right\|_{p}$. Using the integration by parts formula, we obtain

$$
Y_{t}=2 \mathbb{H} \int_{0}^{t}(t-s)^{\mathbb{H}-1 / 2} d M_{s}=-2 \mathbb{H} \int_{0}^{t} M_{s} d(t-s)^{\mathbb{H}-1 / 2} .
$$

The process $Y^{n}$ can be written as

$$
\begin{aligned}
Y_{t}^{n} & =2 \mathbb{H} \sum_{t_{k} \leq t^{*}}\left(t^{*}-t_{k-1}\right)^{\mathbb{H}-1 / 2}\left(M_{t_{k}}^{n}-M_{t_{k-1}}^{n}\right) \\
& =-2 \mathbb{H} \sum_{t_{k} \leq t^{*}} M_{t_{k}}^{n}\left[\left(t^{*}-t_{k}\right)^{\mathbb{H}-1 / 2}-\left(t^{*}-t_{k-1}\right)^{\mathbb{H}-1 / 2}\right] \\
& =-2 \mathbb{H} \int_{0}^{t^{*}} \hat{M}_{s}^{n} d\left(t^{*}-s\right)^{\mathbb{H}-1 / 2}
\end{aligned}
$$


where $\hat{M}_{t}^{n}=M_{\frac{k+1}{n}}$ for $t \in\left[\frac{k}{n}, \frac{k+1}{n}\right)$. Note that (15) still holds if we replace $M^{n}$ by $\hat{M}^{n}$. Now,

$$
\begin{aligned}
\left\|\sup _{t \leq T}\left|Y_{t}-Y_{t}^{n}\right|\right\|_{p} & \leq \operatorname{Const}\left(\left\|\sup _{t \leq T}\left|Y_{t}-Y_{t^{*}}\right|\right\|_{p}+\left\|\sup _{t \leq T}\left|Y_{t^{*}}-Y_{t}^{n}\right|\right\|_{p}\right) \\
& =I_{1}+I_{2} .
\end{aligned}
$$

Combining (5) with (6) and (7) gives

$$
\begin{aligned}
I_{2} & \leq \text { Const }\left\|\sup _{t \leq T}\left|\int_{0}^{t^{*}}\left(M_{s}-\hat{M}_{s}^{n}\right) d\left(t^{*}-s\right)^{\mathbb{H}-1 / 2}\right|\right\|_{p} \\
& \leq \text { Const }\left\|\sup _{t \leq T} E\left|M_{t}-\hat{M}_{t}^{n}\right|\right\|_{p} \\
& \leq \text { Const } \frac{\sqrt{\ln n}}{n^{1-\mathbb{H}}} .
\end{aligned}
$$

To estimate $I_{1}$, we first prove that for any $t_{1}, t_{2} \in[0, T]$ and $q \geq 1$

$$
E\left|Y_{t_{2}}-Y_{t_{1}}\right|^{q} \leq \text { Const }\left|t_{2}-t_{1}\right|^{q(\mathbb{H}-1 / 2)} .
$$

Indeed, by the Burkholder-Davis-Gundy inequality we have

$$
\begin{aligned}
E\left|Y_{t_{2}}-Y_{t_{1}}\right|^{q}= & E \mid \int_{0}^{t_{1}}\left[\left(t_{2}-s\right)^{\mathbb{H}-1 / 2}-\left(t_{1}-s\right)^{\mathbb{H}-1 / 2}\right] d M_{s} \\
& +\left.\int_{t_{1}}^{t_{2}}\left(t_{2}-s\right)^{\mathbb{H}-1 / 2} d M_{s}\right|^{q} \\
\leq & \operatorname{Const}\left(E\left|\int_{0}^{t_{1}}\left[\left(t_{2}-s\right)^{\mathbb{H}-1 / 2}-\left(t_{1}-s\right)^{\mathbb{H}-1 / 2}\right]^{2} d[M]_{s}\right|^{q / 2}\right. \\
& \left.+E\left|\int_{t_{1}}^{t_{2}}\left(t_{2}-s\right)^{2 \mathbb{H}-1} d[M]_{s}\right|^{q / 2}\right) \\
\leq & \operatorname{Const}\left(\left.\left(t_{2}-t_{1}\right)^{q(\mathbb{H}-1 / 2) \mid} \int_{0}^{t_{1}} C s^{1-2 \mathbb{H}} d s\right|^{q / 2}\right. \\
& \left.+\left|\int_{t_{1}}^{t_{2}} C s^{1-2 \mathbb{H}} d s\right|^{q / 2}\right) \\
\leq & \operatorname{Const}\left(t_{2}-t_{1}\right)^{q(\mathbb{H}-1 / 2)}\left|\int_{0}^{T} C s^{1-2 \mathbb{H}} d s\right|^{q / 2} \\
\leq & \operatorname{Const}\left(t_{2}-t_{1}\right)^{q(\mathbb{H}-1 / 2)} .
\end{aligned}
$$

Let $q>\max \left(p,(\mathbb{H}-1 / 2)^{-1}\right)$. By the Kolmogorov continuity theorem,

$$
E\left(\sup _{\substack{t_{1}, t_{2} \leq T \\ t_{1} \neq t_{2}}} \frac{\left|Y_{t_{2}}-Y_{t_{1}}\right|}{\left|t_{2}-t_{1}\right|^{\alpha}}\right)^{q}<+\infty
$$

for $0<\alpha<(\mathbb{H}-1 / 2)-1 / q$. Since $p<q$, from the above it follows that 


$$
I_{1} \leq\left(E \sup _{t \leq T}\left|Y_{t}-Y_{t^{*}}\right|^{q}\right)^{1 / q} \leq \text { Const } \frac{1}{n^{\mathbb{H}-1 / 2-\epsilon}}
$$

for every $\epsilon>0$. By (8) and (9),

$$
\left\|\sup _{t \leq T}\left|Y_{t}-Y_{t}^{n}\right|\right\|_{p} \leq \text { Const } \frac{1}{n^{\mathbb{H}^{*}-\epsilon}} .
$$

It remains to show that $\left\|\sup _{t \leq T}\left|B_{t}^{\mathbb{H}}-B_{t}^{n}\right|\right\|_{p} \leq$ Const $\left\|\sup _{t \leq T}\left|Y_{t}-Y_{t}^{n}\right|\right\|_{p}$. Using once again the integration by parts formula we obtain

$$
B_{t}^{\mathbb{H}}=\int_{0}^{t} s^{\mathbb{H}-1 / 2} d Y_{s}=t^{\mathbb{H}-1 / 2} Y_{t}-\int_{0}^{t} Y_{s} d s^{\mathbb{H}-1 / 2},
$$

and

$$
\begin{aligned}
B_{t}^{n}= & \int_{0}^{t} s^{\mathbb{H}-1 / 2} d Y_{s}^{n}=t^{\mathbb{H}-1 / 2} Y_{t}^{n}-\int_{0}^{t} Y_{s}^{n} d s^{\mathbb{H}-1 / 2} \\
& -\sum_{t_{k} \leq t}\left(Y_{t_{k}}^{n}-Y_{t_{k-1}}^{n}\right)\left(t_{k}^{\mathbb{H}-1 / 2}-t_{k-1}^{\mathbb{H}-1 / 2}\right) .
\end{aligned}
$$

Hence

$$
\begin{aligned}
&\left\|\sup _{t \leq T}\left|B_{t}^{\mathbb{H}}-B_{t}^{n}\right|\right\|_{p} \leq \text { Const }\left(\left|\sup _{t \leq T}\right| t^{\mathbb{H}-1 / 2} Y_{t}-t^{\mathbb{H}-1 / 2} Y_{t}^{n} \mid \|_{p}\right. \\
&+\left\|\sup _{t \leq T}\left|\int_{0}^{t}\left(Y_{s}-Y_{s}^{n}\right) d s^{\mathbb{H}-1 / 2}\right|\right\|_{p} \\
&\left.+\left\|\sup _{t \leq T}\left|\sum_{t_{k} \leq t}\left(Y_{t_{k}}^{n}-Y_{t_{k-1}}^{n}\right)\left(t_{k}^{\mathbb{H}-1 / 2}-t_{k-1}^{\mathbb{H}-1 / 2}\right)\right|\right\|_{p}\right) \\
&=I_{4}+I_{5}+I_{6} .
\end{aligned}
$$

It is easily seen that $I_{4}$ and $I_{5}$ are bounded by Const $\left\|\sup _{t \leq T}\left|Y_{t}-Y_{t}^{n}\right|\right\|_{p}$. To estimate $I_{6}$ we observe first that

$$
\begin{aligned}
\sup _{t_{k} \leq T}\left|Y_{t_{k}}^{n}-Y_{t_{k-1}}^{n}\right| & =\sup _{t \leq T}\left|Y_{t}^{n}-Y_{t-}^{n}\right|=\sup _{t \leq T}\left|\left(Y_{t}^{n}-Y_{t-}^{n}\right)-\left(Y_{t}-Y_{t-}\right)\right| \\
& \leq 2 \sup _{t \leq T}\left|Y_{t}-Y_{t}^{n}\right| .
\end{aligned}
$$

Hence

$$
I_{6} \leq T^{\mathbb{H}-1 / 2}\left\|\sup _{t_{k} \leq T}\left|Y_{t_{k}}^{n}-Y_{t_{k-1}}^{n}\right|\right\|_{p} \leq \mathrm{Const}\left\|\sup _{t \leq T}\left|Y_{t}-Y_{t}^{n}\right|\right\|_{p}
$$

which completes the proof. 


\section{Approximation of Prices for Path Dependent Options}

The method described in the previous section can be used to approximate the price evolution process $S$. Let $S^{n}$ be the approximation of $S$ defined by

$$
S_{t}^{n}=S_{0} e^{\mu t+\sigma B_{t}^{n}}
$$

for all $t \in[0, T]$. The following result is a consequence of Theorem 1

Theorem 2. For any $p \geq 1, \epsilon>0$ and $T \in \mathbb{R}^{+}$

$$
\left\|\sup _{t \leq T}\left|S_{t}-S_{t}^{n}\right|\right\|_{p}=\mathcal{O}\left(\frac{1}{n^{\mathbb{H}^{*}-\epsilon}}\right) .
$$

Proof. Without loss of generality we may and will assume that $S_{0}=1, \mu=$ $0, \sigma=1$. Observe first that by the Schwarz inequality,

$$
\begin{aligned}
\left\|\sup _{t \leq T}\left|S_{t}-S_{t}^{n}\right|\right\|_{p} & =\left\|\sup _{t \leq T}\left|e^{B_{t}^{\mathbb{H}}}\left[e^{\left(B_{t}^{\mathbb{H}}-B_{t}^{n}\right)}-1\right]\right|\right\|_{p} \\
& \leq\left\|\sup _{t \leq T}\left|e^{B_{t}^{\mathbb{H}}}\right|\right\|_{2 p}\left\|\sup _{t \leq T}\left|e^{\left(B_{t}^{\mathbb{H}}-B_{t}^{n}\right)}-1\right|\right\|_{2 p} .
\end{aligned}
$$

Since $\left|e^{x}-1\right| \leq|x| e^{|x|}$, we have

$$
\begin{aligned}
\left\|\sup _{t \leq T}\left|e^{\left(B_{t}^{\mathbb{H}}-B_{t}^{n}\right)}-1\right|\right\|_{2 p} & \leq\left\|\sup _{t \leq T}\left|B_{t}^{\mathbb{H}}-B_{t}^{n}\right| \cdot e^{\left|B_{t}^{\mathbb{H}}-B_{t}^{n}\right|}\right\|_{2 p} \\
& \leq\left\|\sup _{t \leq T}\left|B_{t}^{\mathbb{H}}-B_{t}^{n}\right|\right\|_{4 p}\left\|\sup _{t \leq T} e^{\left|B_{t}^{\mathbb{H}}-B_{t}^{n}\right|}\right\|_{4 p} .
\end{aligned}
$$

By Theorem 1 .

$$
\left\|\sup _{t \leq T}\left|B_{t}^{\mathbb{H}}-B_{t}^{n}\right|\right\|_{4 p}=\mathcal{O}\left(\frac{1}{n^{\mathbb{\mathbb { H } ^ { * }}-\epsilon}}\right),
$$

so it remains to show that $\left\|\sup _{t \leq T} e^{B_{t}^{\mathrm{IH}}}\right\|_{8 p}<+\infty$ and $\sup _{n}\left\|\sup _{t \leq T} e^{B_{t}^{n}}\right\|_{8 p}<$ $+\infty$. Novikov and Valkeila [3] proved that $\sup _{t \leq T}\left|B_{t}^{\mathbb{H}}\right| \leq C \sup _{t \leq T}\left|M_{t}\right|$ for some constant $C>0$. Therefore, by the Doob submartingale inequality,

$$
\begin{aligned}
\left\|\sup _{t \leq T} e^{B_{t}^{\mathbb{H}}}\right\|_{8 p} & =\left(E e^{\sup _{t \leq T}\left(8 p\left|B_{t}^{\mathbb{H}}\right|\right)}\right)^{1 /(8 p)} \\
& \leq\left(E \sup _{t \leq T} e^{8 p C\left|M_{t}\right|}\right)^{1 /(8 p)} \\
& \leq\left(4 E e^{8 p C\left|M_{T}\right|}\right)^{1 /(8 p)}<+\infty
\end{aligned}
$$

because $M$ is a Gaussian martingale. Finally, by (11) and (7),

$$
\begin{aligned}
\sup _{t \leq T}\left|B_{t}^{n}\right| \leq & T^{\mathbb{H}-1 / 2} \sup _{t \leq T}\left|Y_{t}^{n}\right|+T^{\mathbb{H}-1 / 2} \sup _{t \leq T}\left|Y_{t}^{n}\right| \\
& +T^{\mathbb{H}-1 / 2} 2 \sup _{t \leq T}\left|Y_{t}^{n}\right| \leq 4 T^{\mathbb{H}-1 / 2} \sup _{t \leq T}\left|Y_{t}^{n}\right| \\
\leq & 8 \mathbb{H} T^{2 \mathbb{H}-1} \sup _{t \leq T}\left|M_{t}^{n}\right| .
\end{aligned}
$$


Therefore, similarly to (12) we get

$$
\begin{aligned}
\sup _{n}\left\|\sup _{t \leq T} e^{B_{t}^{n}}\right\|_{8 p} & \leq \sup _{n}\left(4 E e^{64 p \mathbb{H} T^{2 \mathbb{H}-1}\left|M_{T}^{n}\right|}\right)^{1 /(8 p)} \\
& \leq\left(4 E e^{64 p \mathbb{H} T^{2 \mathbb{H}-1}\left|M_{T}\right|}\right)^{1 /(8 p)}<+\infty
\end{aligned}
$$

and the proof is complete.

Definition 3. We say that an operator $H: C\left([0, T], \mathbb{R}^{+}\right) \rightarrow \mathbb{R}$ is Lipschitz if there exists $L>0$ such that for any $x, y \in C\left([0, T], \mathbb{R}^{+}\right)$

$$
|H(x)-H(y)| \leq L \cdot \sup _{t \leq T}\left|x_{t}-y_{t}\right|
$$

From Theorem 1 and Definition 3 we obtain immediately the following corollary.

Corollary 1. If $H: C\left([0, T], \mathbb{R}^{+}\right) \rightarrow \mathbb{R}$ is Lipschitz then for any $p \geq 1, \epsilon>0$

$$
\left\|H(S)-H\left(S^{n}\right)\right\|_{p}=\mathcal{O}\left(\frac{1}{n^{\mathbb{H}^{*}-\epsilon}}\right) .
$$

By choosing appropriately a Lipschitz operator $H$ we obtain interesting examples of path dependent options of the form $f_{T}=H(S)$.

Example 1 (Floating strike lookback call option).

In this case $H(S)=S_{T}-\inf _{t \leq T} S_{t}$. Note that for any $x, y \in C\left([0, T], \mathbb{R}^{+}\right)$

$$
\begin{aligned}
|H(x)-H(y)| & =\left|\left(x_{T}-y_{T}\right)+\left(\inf _{t \leq T} y_{t}-\inf _{t \leq T} x_{t}\right)\right| \\
& \leq 2 \sup _{t \leq T}\left|x_{t}-y_{t}\right| .
\end{aligned}
$$

Example 2 (Fixed strike lookback call option with the strike price $K$ ).

Here $H(S)=\left(\sup _{t \leq T} S_{t}-K\right)^{+}$and for any $x, y \in C\left([0, T], \mathbb{R}^{+}\right)$

$$
|H(x)-H(y)| \leq\left|\sup _{t \leq T} x_{t}-\sup _{t \leq T} y_{t}\right| \leq \sup _{t \leq T}\left|x_{t}-y_{t}\right| .
$$

Example 3 (Asian call option with the strike price $K$ and continuous arithmetic averaging).

For such options

$$
H(S)=\left(\frac{1}{T} \int_{0}^{T} S_{t} d t-K\right)^{+}
$$

and it is easily seen that 


$$
\begin{aligned}
|H(x)-H(y)| & =\left|\left(\frac{1}{T} \int_{0}^{T} x_{t} d t-K\right)^{+}-\left(\frac{1}{T} \int_{0}^{T} y_{t} d t-K\right)^{+}\right| \\
& \leq\left|\frac{1}{T} \int_{0}^{T}\left(x_{t}-y_{t}\right) d t\right| \leq \sup _{t \leq T}\left|x_{t}-y_{t}\right|
\end{aligned}
$$

for any $x, y \in C\left([0, T], \mathbb{R}^{+}\right)$.

\section{Numerical Results}

The method described in Sections 2 and 3 we have applied to evaluate prices of the lookback and Asian options. We have simulated 100000 trajectories of $S$ with step-size $1 / 1000$.

Table 1 gives prices of the floating strike lookback options (LB1), fixed strike lookback options with the strike price $K_{1}=5$ (LB2) and Asian options with the strike price $K 2=5$ (AS) for different choises of the Hurst index $\mathbb{H}$. The initial asset price $S_{0}$ is set equal to 5 , parameters $r$ and $\sigma$ are equal 0.1 and 0.3 respectively.

Table 1. The prices of LB1, LB2 and AS $\left(S_{0}=5, K_{1}=5, K_{2}=5, \sigma=0.3, r=0.1\right.$, $T=1)$.

\begin{tabular}{lcccc}
\hline H & 0.6 & 0.7 & 0.8 & 0.9 \\
\hline LB1 & 1.1381 & 1.0288 & 0.9399 & 0.8861 \\
LB2 & 1.2727 & 1.1177 & 1.0013 & 0.9138 \\
AS & 0.4464 & 0.4358 & 0.4234 & 0.4207 \\
\hline
\end{tabular}

\section{References}

1. Kwok Y.K.: Mathematical Models of Financial Derivatives. Springer, Singapore (1998)

2. Norros I., Valkeila E., Virtamo J.: An elementary approach to a Grisanov formula and other analytical results on fractional Brownian motions. Bernoulli 5(4) (1999) 571-587

3. Novikov A., Valkeila E.: On some maximal inequalities for fractional Brownian motions. Statistics \& Probability Letters 44 (1999) 47-54

4. Shiryaev A.N.: On Arbitrage and Replication for Fractal Models. Research Report 20, MaPhySto, Centre for Mathematical Physics and Stochastics, September (1998)

5. Słomiński L.: Euler's approximations of solutions of SDEs with reflecting boundary. Stochastics Processes and their Applications 94 (2001) 317-337

6. Valkeila E.: On some properties of geometric fractional Brownian motions. Preprint (1998) 